\title{
Pain Specialists Awareness of Topical Analgesics and Their Use in Pain Control: Survey Results
}

\author{
Joseph V. Pergolizzi1 ${ }^{12^{*}}$, Robert B. Raffa ${ }^{3}$, Robert Taylor ${ }^{4}$, Charles Fleischer ${ }^{4}$, Gianpietro Zampogna ${ }^{4}$, \\ Lynn Nopper ${ }^{5}$, Frank DiLorenzo ${ }^{5}$, Clay M. Duval ${ }^{4}$, Alana N. Edun ${ }^{4}$, Jasmine Salamara $^{5}$
}

\author{
${ }^{1}$ Department of Medicine, Johns Hopkins University School of Medicine, Baltimore, MD, USA \\ ${ }^{2}$ Department of Pharmacology, Temple University School of Medicine, Philadelphia, PA, USA \\ ${ }^{3}$ Department of Pharmaceutical Sciences, Temple University School of Pharmacy, Philadelphia, PA, USA \\ ${ }^{4}$ NEMA Research Inc., Bonita Springs, FL, USA \\ ${ }^{5}$ Healthy Directions, Bethesda, MD, USA
}

*Corresponding author: Joseph V. Pergolizzi, Department of Medicine, Johns Hopkins University School of Medicine, Baltimore, USA, Department of Pharmacology, Temple University School of Medicine, Philadelphia, PA, USA, Tel: 239.597.3662/239.908.4442, Fax: 239.908.4432; E-mail: jpjmd@msn.com

\begin{abstract}
Background: Over-the-counter (OTC) topical analgesics are an important part of the armamentarium for pain management, but it was not clear if and to what extent prescribers discussed the option of topical analgesics with their patients.
\end{abstract}

Objective: The objective was to assess the extent to which pain specialists were aware of topical analgesics and how often and under what circumstances they recommended topical pain relievers to their patients to help control painful symptoms.

Methods: The authors conducted a convenience-sample survey at the PAINWeek ${ }^{\circledR}$ meeting in Las Vegas in September 2014. The survey was distributed during the conference and there were 81 respondents (healthcare providers).

Results: Eight-five percent of all respondents ( $100 \%$ of pain physicians) indicated that they recommended OTC topical analgesics to patients and $81.5 \%$ said they had no concerns about the safety and tolerability of such products. When asked if topical analgesics could be a good first step in relieving muscle and joint pain, $98.8 \%$ of all respondents and $100 \%$ of pain specialist physicians expressed agreement.

Conclusion: OTC topical analgesics represent an important option for treating muscle and joint pain. They may offer specific advantages over other pain relievers in that they do not require high serum concentrations of the active agent and therefore may be associated with fewer side effects. OTC topical analgesic products can be easily obtained, self-administered, and allow for improved accessibility. Healthcare professionals specializing in pain are aware of topical analgesics and consider them safe, effective, and a good first-step product in for treating musculoskeletal pain. Clinically tested OTC topical analgesics can be used with confidence as a first-line treatment for acute musculoskeletal pain.
Received date: February 18, 2016

Accepted date: March 30, 2016

Published date: March 31, 2016

Citation: Pergolizzi, J.V., et al. Pain Specialists Awareness of Topical Analgesics and Their Use in Pain Control: Survey Results. (2016) J Anesth Surg 3(1): 114- 118.

DOI: $10.15436 / 2377-1364.16 .034$

Keywords: Topical analgesics; Topical pain relievers; Chronic pain; Acute pain; Musculoskeletal pain; Arthritis

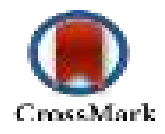

\section{Introduction}

Pain is inherently subjective and although it can be objectively measured using any number of validated tools ${ }^{[1-3]}$, it often requires an individualized approach. According to the International Association for the Study of Pain (IASP), pain has been defined as an unpleasant sensory and emotional experience associated with actual or potential tissue damage, or described by the patients in terms of such damage ${ }^{[4]}$. Pain can be described in a number of ways: in terms of intensity (mild, moderate, severe, and very severe), duration (acute versus chronic), mechanism (neuropathic, visceral, nociceptive), and underlying disease (such as cancer pain). These factors may influence treatment choices, but analgesic products require prescribers to balance associated benefits against 
potential risks. Current approaches to pain control have not always offered satisfactory results. IASP states that some of the essential diagnostic criteria for acute musculoskeletal pain are pain and tenderness in a particular region in the absence of any serious pathology.

For acute pain, the goal is safe, effective, and rapid analgesia that allows mobilization and healing to take place ${ }^{[5]}$.

Both short and long-term use of many pharmacotherapeutic regimens has been associated with safety issues, tolerability, and, in the case of opioids, tolerance, hyperalgesia, and misuse ${ }^{[6-8]}$. Toxicity issues have been raised with acetaminophen ${ }^{[9,10]}$, tolerability with NSAIDs ${ }^{[11]}$, and recent safety concerns with NSAID with respect to cardiovascular risks ${ }^{[12,13]}$. This leaves many pain patients with limited therapeutic options and inadequate pain control $^{[14,15]}$. As international organizations declare that pain relief is a fundamental human right ${ }^{[16]}$, physicians must balance concerns about unrelieved pain against safety issues.

Topical analgesic products offer a number of potential advantages for pain control in some patient populations. Unlike oral analgesics, topical products are designed not to be systematically absorbed and therefore may have less potential to produce adverse systemic effects. This can be of particular importance to athletes who may seek pain relief without concomitant systemic side effects such as drowsiness. Topical products can deliver medication directly to the site of pain with minimal adverse effects. Topical products (applied to the skin surface at or around the painful area) differ from transdermal products (deliver the drug through the skin and result in systemic exposure to the drug) in that topical agents increase drug concentration at the application site with minimal systemic uptake and therefore low serum concentration.

Topical nonsteroidal anti-inflammatory drugs (NSAIDs), such as diclofenac and ibuprofen, require a prescription in the United States and include the same black-box warnings as all NSAIDs $^{[17]}$. Other topical products available over-the-counter (OTC) are more readily available to consumers but there may be a lack scientific evidence in support of their effectiveness and safety which makes it difficult for prescribers to recommend the right product for a particular patient. In fact, many healthcare professionals do not recommend or prescribe topical analgesics to their pain patients despite their potential advantages and utility.

The purpose of our survey was to determine if healthcare providers involved in the care of pain patients were aware of topical products including OTC preparations, what they thought of them, and how they incorporated them into their practice. A better understanding of the knowledge attitudes and perceptions of healthcare providers towards the use of OTC topical analgesics would provide clarity into their potential role an option to manage acute and chronic pain.

\section{Methods}

During a United States meeting of pain specialists (PAINWeek ${ }^{\circledR}$ 2014, Las Vegas, Nevada) a six-item paper survey was distributed among attendees regardless of their role in pain management or their pain management experience. This was a convenience sample designed to gather information about the knowledge of these healthcare providers related to topical analgesics, their attitudes toward the use of these products, and how topical analgesics were used in their practice. No respondents were compensated for their participation in the survey.

The full text of the survey appears in the Appendix. The text of the survey distributed at the PAINWeek ${ }^{\circledR}$ meeting.

\begin{tabular}{|c|}
\hline Pain Week Attendee Survey \\
\hline $\begin{array}{l}\text { Are you a pain management physican? } \\
\text { [ Yes } \\
\text { [ No }\end{array}$ \\
\hline 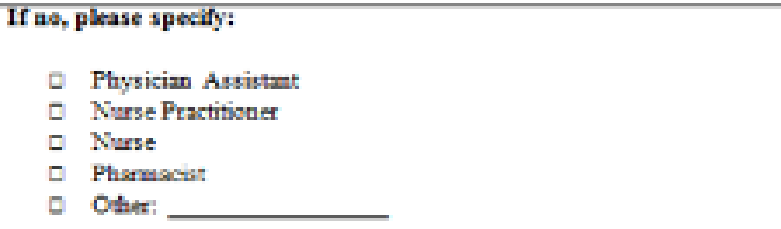 \\
\hline $\begin{array}{l}\text { Do yea recommend the use of OTC tapicals to your parients? } \\
\text { o Yes } \\
\text { D No }\end{array}$ \\
\hline Which product do you recommend, or do yea think they use? \\
\hline $\begin{array}{l}\text { Do you have a concern about patients esing OrC paie creams? } \\
\text { Y Yes } \\
\text { D No }\end{array}$ \\
\hline $\begin{array}{l}\text { Do yea think topical analgesics could be a good first step in relieving } \\
\text { musele and joint pain? } \\
\text { [ Yes } \\
\text { 口 No }\end{array}$ \\
\hline
\end{tabular}

\section{Results}

A total of 81 healthcare providers took the survey but not all respondents answered all of the questions. Respondents represented a diverse range of healthcare specialties, including physicians, physician assistants, nurse practitioners, nurses, pharmacists, acupuncturists, oncologists, and others. See Table 1.

Table 1: Specialties of respondents who participated in the survey; not all respondents provided this information.

\begin{tabular}{|l|l|}
\hline \multicolumn{2}{|c|}{ Specialty $(n=79)$} \\
\hline Nurse practitioner & $31(39 \%)$ \\
\hline Pain management physician & $21(27 \%)$ \\
\hline Pharmacist & $8(10 \%)$ \\
\hline Nurse & $8(10 \%)$ \\
\hline Physician assistant & $6(8 \%)$ \\
\hline Family medicine & $2(3 \%)$ \\
\hline Physical therapist & $1(1 \%)$ \\
\hline Acupuncturist & $1(1 \%)$ \\
\hline Oncologist & $1(1 \%)$ \\
\hline Total & $79(100 \%)$ \\
\hline
\end{tabular}


Approximately $85 \%$ of healthcare providers $(n=69)$ surveyed indicated that they currently recommended overthe-counter (OTC) topical analgesic products to their patients. Among pain management physicians, 100\% recommended OTC topical analgesics to their patients. (See Figure1) Many respondents could name specific OTC products.

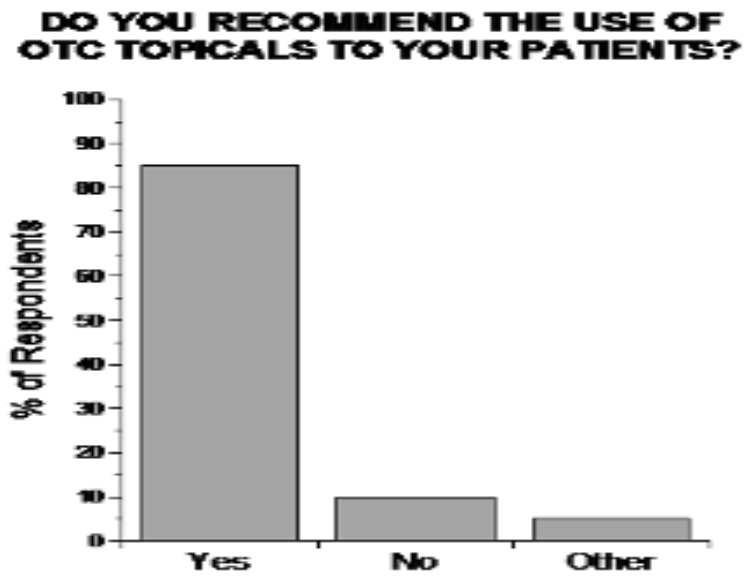

Figure 1: Respondents' recommendations to their patients regarding OTC topical analgesics.

Topical analgesic products were generally considered to be safe. The majority of respondents $(81.5 \%)$ said they were not concerned about issues of safety and tolerability when their patients used OTC topical analgesics. Topical administration reduces systemic exposure to active agents.

Nearly all respondents $(98.8 \%)$ stated that topical analgesic products could be a good first step in relieving muscle and joint pain. Among pain specialists, $100 \%$ thought topical analgesics were a good first-step product. See Figure 2.

\section{DO YOU THINK TOPICAL ANALGE SICS} COULD BE A GOOD FIR ST STEP IN RELIEVING MU SCLE AND JOINT PAIN?

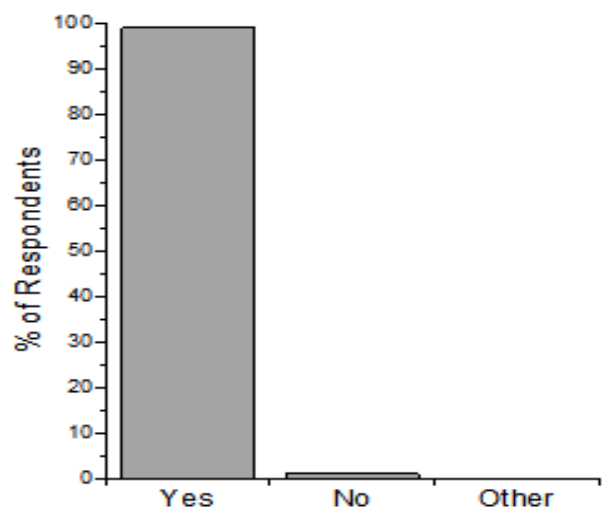

Figure 2: Respondents' attitudes about the use of topical analgesics as first product for painful musculoskeletal conditions.

\section{Discussion}

Topical analgesic products are well known and may provide reasonable safe, effective first-step relief for certain types of musculoskeletal pain ${ }^{[18]}$. Indeed, they may offer specific advantages to pain control for chronic, refractory pain syndromes. While topical analgesic products may be used alone, they can also be an important component in multimodal pain control regimens. Multimodal pain management strategies incorporate nonpharmacological and pharmacological options into the pain management plan and attempt to address pain by various means. Thus, it may be appropriate for a patient to treat pain with lifestyle modifications and a topical agent and possibly an oral agent as well.

Applied at or near the painful site, topical agents increase drug concentration at the application site but their low systemic uptake means there is negligible serum concentration of the active agent ${ }^{[19]}$. Topical agents thus work at the peripheral rather than central targets and are thus most suitable in painful syndromes with a strong peripheral component (for example, osteoarthritis pain). Topical analgesics can also be effective in treating refractory pain associated with nerve damage ${ }^{[18]}$.

OTC topical analgesics rely on NSAIDs, salicylates, local anesthetics (such as lidocaine), capsaicin, menthol, and camphor, but other agents may be used as well, such as tricyclic antidepressants, ketamine, anticonvulsants, or combinations of drugs $^{[19]}$. In a meta-analysis of safety data, topical NSAIDs show good safety results ${ }^{[20]}$. In a study of topical diclofenac sodium $1 \%$ gel in geriatric patients with pain associated with osteoarthritis $(n=947)$, patient subgroups with hypertension, diabetes mellitus, and cerebrovascular or cardiovascular disease could use the topical product as safely as those without those comorbidities $^{[21]}$. A meta-analysis of studies on high-concentration topical capsaicin for chronic neuropathic pain found it to be safe and effective ${ }^{[22]}$, with more equivocal results for topical lidocaine in the treatment of neuropathic pain ${ }^{[23]}$.

Many OTC topical analgesics do not have extensive clinical studies to support their use and this creates uncertainly for practitioners when making recommendations to patients. Menthol is a commonly used agent in OTC topical analgesic preparations. The mechanism of action is not fully understood but is believed to be multifaceted involving both a counter-irritant effect as it relates to the "gate theory" ${ }^{[24]}$ and an effect on specific transient receptor potential (TRPM8) receptors ${ }^{[25]}$. The counter-irritant effect results in an inability to perceive a painful stimulus and the modulation of TRPM8 produces a cooling effect (cold analgesia) that results in pain relief. Studies have shown that menthol in topical products may cool the skin at higher concentrations (typically $4.6 \%$ to $10 \%)^{[26]}$. Topical menthol was found to show potential as a novel pain reliever for chemotherapy-induced neuropathic pain in a proof-of-concept study evaluating $1 \%$ menthol cream $^{[27]}$. Topical menthol was also shown in a study $(\mathrm{n}=645)$ to significantly reduce pain associated with carpal tunnel syndrome compared to placebo ${ }^{[28]}$. A methyl salicylate and menthol patch in a single eight-hour application provided muscle strain patients with significantly better pain control than placebo $(n=208)^{[29]}$. The minimum effective dose of menthol is not known.

Novel topical menthol preparations with combined active excipients may potentially create unexpected improvements in pain relief. A novel low-dose menthol-based OTC topical analgesic preparation (OxyRub ${ }^{\mathrm{TM}}$, Healthy Directions) has been recently introduced in the market. The product has been clinically tested for safety and effectiveness in treating acute pain ${ }^{[30]}$. Taylor et al. conducted a study for the temporary relief of acute pain, comparing menthol plus oxygenated glycerol trimester oil topical preparation to menthol single-entity preparation and re- 
ported that the combined product offered significantly improved pain relief versus mentholated cream alone ${ }^{[31]}$.

Due to the paucity in the literature of head-to-head comparative safety and efficacy studies of two or more topical analgesics, patients and healthcare providers must make product choices based on anecdotal information or marketing-related influences. A comprehensive review of six studies ( $>600$ subjects) found that topical NSAID analgesics could be considered comparably effective as oral NSAIDs in relieving knee pain associated with osteoarthritis, but with fewer serious adverse events associated with the topical NSAID products ${ }^{[32]}$. It is unlikely that consumers are aware of such studies and ongoing research.

One important issue in consideration of any topical product must be the carrier. Permeation enhancers can be added to some topical agents to enhance skin permeation and analgesic efficacy ${ }^{[33]}$. Rosemary essential oil has been used to enhance transdermal absorption of sodium diclofenac gel ${ }^{[34]}$. Thymol has been employed to help enhance absorption of meloxicam through the $\operatorname{skin}^{[35]}$. Hydrophilic gels and terpenes have been used in studies to test etodolac absorption through the skin ${ }^{[36]}$. The role of excipients in topical drug distribution is currently any area of study but much remains to be elucidated. Nanospheres dispersed in gels have also been suggested to optimize transdermal delivery of liphophilic drugs ${ }^{[37]}$. In some cases, these permeation enhancers appear to offer positive benefits for transdermal drug delivery ${ }^{[38]}$.

The study results showed that healthcare providers, particularly pain management specialists, see tremendous value in the use of topical analgesics for the management of acute pain in patients, even though supporting data are limited.

\section{Conclusion}

OTC topical analgesics may play an important role in addressing the epidemic of acute pain, particularly since many of the currently available oral pharmacological therapies are associated with safety, toxicity, abuse potential, and tolerability issues that can limit their use. Topical analgesics deliver the active agent directly to the painful site and do produce systemic adverse events, although OTC or prescription topical analgesics are unfortunately not routinely recommended to appropriate patients. Healthcare providers perceive a benefit to using topical analgesics and most recommend them as a first line of therapy. Our survey showed that $100 \%$ of the pain management physicians would recommend OTC topical analgesics to their patients. Most respondents said they were not concerned with the safety and tolerability of OTC topical analgesics (81.5\%) and that they were a good first step in relieving muscle and joint pain $(98.8 \%)$ despite that fact there is limited information in the medical literature to support OTC analgesics. Novel OTC analgesics preparations that have been clinically tested and demonstrated reasonable safety and efficacy present a valid option for the temporarily relief of minor aches and pains of muscles and joints. More studies on OTC topical analgesics are needed, especially studies comparing products to each other for safety and efficacy.

Acknowledgements: This manuscript was prepared with medical writing and editing support by Jo Ann LeQuang of LeQ Medical whose services were paid by the authors. The authors thank PAINWeek ${ }^{\circledR}$ and survey respondents for their participation.
Conflict of Interest: Lynn Nopper, Jasmine Salamara, and Frank Dilorenzo are employees of Healthy Directions. Healthy Directions distributes Dr. Pergolizzi's topical pain reliever, OxyRub $^{\mathrm{TM}}$. Dr. Taylor and Dr. Zampognaare employees of NEMA Research. NEMA Research received funding for the preparation of this abstract from Healthy Directions.

\section{References}

1. Caraceni, A. Evaluation and assessment of cancer pain and cancer pain treatment. (2001) Acta Anaesthesiol Sc 45(9): 10671075.

2. Caraceni, A., Cherny, N., Fainsinger, R., et al. Pain measurement tools and methods in clinical research in palliative care: recommendations of an Expert Working Group of the European Association of Palliative Care. (2002) J pain and symptom manage 23(3): 239-255.

3. Williamson A, Hoggart B. Pain: a review of three commonly used pain rating scales. (2005) J Clin Nurs 14(7): 798-804.

4. International Association for the Study of Pain. (2012) Pain Terms, IASP Taxonomy.

5. Arendt-Nielsen, L., Fernandez-de-Las-Penas, C., Graven-Nielsen, T. Basic aspects of musculoskeletal pain: from acute to chronic pain. (2011) J Man Manip Ther 19(4): 186-193.

6. Ahlbeck, K. Opioids: a two-faced Janus. (2011) Curr Med Res Opin 27(2): 439-448.

7. Ling, W., Mooney, L., Hillhouse, M. Prescription opioid abuse, pain and addiction: clinical issues and implications. (2011) Drug Alcohol Rev 30(3): 300-305.

8. Chang, G., Chen, L., Mao, J. Opioid tolerance and hyperalgesia. (2007) Med Clin North Am 91(2): 199-211.

9. Brune, K., Hinz, B., Otterness, I. Aspirin and acetaminophen: should they be available over the counter? (2009) Curr Rheumatol Rep 11(1): 36-40.

10. FDA. Acetaminophen Toxicity. (2013) Drug Safety and Availability.

11. Biskupiak, J.E., Brixner, D.I., Howard, K., et al. Gastrointestinal complications of over-the-counter nonsteroidal antiinflammatory drugs. (2006) Journal of pain \& palliative care pharmacotherapy 20(3): 7-14.

12. Pham, T.T., Miller, M.J., Harrison, D.L., et al. Cardiovascular disease and non-steroidal anti-inflammatory drug prescribing in the midst of evolving guidelines. (2013) J Eval Clin Pract 19(6): 1026-1034.

13. Adams, R.J., Appleton, S.L., Gill, T.K., et al. Cause for concern in the use of non-steroidal anti-inflammatory medications in the community--a population-based study. (2011) BMC Fam Pract 12:70.

14. Gandhi, K., Heitz, J.W., Viscusi, E.R. Challenges in acute pain management. (2011) Anesthesiol Clin 29(2): 291-309.

15. Daher, M. Pain relief is a human right. (2010) Asian Pac J Cancer Prev 1: 97-101.

16. Brennan, F., Carr, D.B., Cousins, M. Pain management: a fundamental human right. (2007) Anesth Analg 105(1): 205-221.

17. Bradford, W.D., Kleit, A.N. Impact of FDA Actions, DTCA, and Public Information on the Market for Pain Medication. (2015) Health Econ 24(7): 859-875.

18. Argoff, C.E. Topical analgesics in the management of acute 
and chronic pain. Introduction. (2013) Postgrad Med $125(4$ Suppl 1): 3-6.

19. Anitescu, M., Benzon, H.T., Argoff, C.E. Advances in topical analgesics. (2013) Curr Opin Anaesthesiol 26(5): 555-561.

20. Barkin, R.L. Topical Nonsteroidal Anti-Inflammatory Drugs: The Importance of Drug, Delivery, and Therapeutic Outcome. (2015) Am J Ther 22(5): 388-407.

21. Peniston, J.H., Gold, M.S., Wieman, M.S., et al. Long-term tolerability of topical diclofenac sodium $1 \%$ gel for osteoarthritis in seniors and patients with comorbidities. (2012) Clin Interv Aging 7: 517-523.

22. Derry, S., Sven-Rice, A., Cole, P., et al. Topical capsaicin (high concentration) for chronic neuropathic pain in adults. (2013) Cochrane Database Syst Rev 2: CD007393.

23. Mick, G., Correa-Illanes, G. Topical pain management with the $5 \%$ lidocaine medicated plaster--a review. (2012) Curr Med Res Opin 28(6): 937-951.

24. Melzack R. From the gate to the neuromatrix. Pain management nursing. (1999) Journal of the American Society of Pain Management Nurses 82 (Suppl): S121-S126.

25. Bodding, M., Wissenbach, U., Flockerzi, V. Characterisation of TRPM8 as a pharmacophore receptor. (2007) Cell Calcium 42(6): 618-628.

26. Lasanen, R., Julkunen, P., Airaksinen, O., et al. Menthol concentration in topical cold gel does not have significant effect on skin cooling. (2016) Skin research and technology: official journal of International Society for Bioengineering and the Skin (ISBS) and International Society for Digital Imaging of Skin (ISDIS) and International Society for Skin Imaging (ISSI).

27. Fallon, M.T., Storey, D.J., Krishan, A., et al. Cancer treatment-related neuropathic pain: proof of concept study with menthol--a TRPM8 agonist. (2015) Support Care Cancer 23(9): 2769-2777.

28. Sundstrup, E., Jakobsen, M.D., Brandt, M., et al. Acute effect of topical menthol on chronic pain in slaughterhouse workers with carpal tunnel syndrome: triple-blind, randomized placebo-controlled trial. (2014) Rehabil Res Pract 310913.

29. Higashi, Y., Kiuchi, T., Furuta, K. Efficacy and safety pro- file of a topical methyl salicylate and menthol patch in adult patients with mild to moderate muscle strain: a randomized, double-blind, parallel-group, placebo-controlled, multicenter study. (2010) Clin Ther 32(1): 34-43.

30. Pergolizzi, J.V., Pappagallo, M., Raffa, R.B., et al. Preliminary observations of a novel topical oil with analgesic properties for treatment of acute and chronic pain syndromes. (2010) Pain Pract 10(3): 201-213.

31. Taylor, R., Gan, T.J., Raffa, R.B., et al. A randomized, double-blind comparison shows the addition of oxygenated glycerol triesters to topical mentholated cream for the treatment of acute musculoskeletal pain demonstrates incremental benefit over time. (2012) Pain Pract 12(8): 610-619.

32. Klinge, S.A., Sawyer, G.A. Effectiveness and safety of topical versus oral nonsteroidal anti-inflammatory drugs: a comprehensive review. (2013) Phys Sportsmed 41(2): 64-74.

33. Al-Suwayeh, S.A., Taha, E.I., Al-Qahtani, F.M., et al. Evaluation of skin permeation and analgesic activity effects of carbopol lornoxicam topical gels containing penetration enhancer. (2014) ScientificWorld Journal 127495.

34. Akbari, J., Saeedi, M., Farzin, D., et al. Transdermal absorption enhancing effect of the essential oil of Rosmarinus officinalis on percutaneous absorption of $\mathrm{Na}$ diclofenac from topical gel. (2015) Pharm Biol 53(10): 1442-1447.

35. Mohammadi-Samani, S., Yousefi, G., Mohammadi, F., et al. Meloxicam transdermal delivery: effect of eutectic point on the rate and extent of skin permeation. (2014) Iran J Basic Med Sci 17(2): 112-118.

36. Tas, C., Ozkan, Y., Okyar, A., et al. In vitro and ex vivo permeation studies of etodolac from hydrophilic gels and effect of terpenes as enhancers. (2007) Drug Deliv 14(7): 453-459.

37. Batheja, P., Sheihet, L., Kohn, J., et al. Topical drug delivery by a polymeric nanosphere gel: Formulation optimization and in vitro and in vivo skin distribution studies. (2011) J Control Release 149(2): 159-167.

38. El-Setouhy, D.A., El-Ashmony, S.M. Ketorolac trometamol topical formulations: release behaviour, physical characterization, skin permeation, efficacy and gastric safety. (2010) J Pharm Pharmacol 62(1): 25-34.
Ommega Online Publishers

Journal Title: Journal of Anesthesia and Surgery (JAS)

Journal Short Name: J Anesth Surg
Journal ISSN: 2377-1364

E-mail: anestheisa@ommegaonline.com

Website: www.ommegaonline.org 